\title{
EFEITO DE INJÚRIAS MECÂNICAS NA FIRMEZA E COLORAÇÃO DE GOIABAS DAS CULTIVARES PALUMA E PEDRO SATO ${ }^{1}$
}

\author{
BEN-HUR MATTIUZ² \& JOSÉ FERNANDO DURIGAN ${ }^{3}$
}

\begin{abstract}
RESUMO - O objetivo deste trabalho foi avaliar o efeito das injúrias mecânicas por impacto, compressão ou corte na firmeza e coloração de goiabas 'Paluma' e 'Pedro Sato', colhidas no estádio de maturação “de vez” e armazenadas sob condições de ambiente. $\mathrm{Na}$ injúria por impacto, os frutos foram deixados cair, em queda livre, de uma altura de 1,20 m, sofrendo dois impactos, em lados opostos da porção equatorial do fruto. Na injúria por compressão, os frutos foram submetidos a um peso de $3 \mathrm{~kg}$, por 15 minutos. Para a injúria por corte, foram efetuados dois cortes, no sentido longitudinal dos frutos, de exatamente $30 \mathrm{~mm}$ de comprimento por $2 \mathrm{~mm}$ de profundidade. Os frutos injuriados foram colocados em bandejas de isopor e armazenados sob condições de ambiente $\left(23,4 \pm 1^{\circ} \mathrm{C}\right.$, $62 \pm 6 \%$ UR). A firmeza dos frutos submetidos ao impacto e compressão foi calculada pela relação peso(N)/área injuriada (m2). A evolução da coloração foi feita através de leituras diárias em reflectômetro Minolta CR 200b, procurando comparar a coloração da área lesionada com a da área não lesionada do mesmo fruto. Com relação à injúria por compressão, não se detectou diferença significativa entre as cultivares testadas, mas com relação ao impacto, os frutos da 'Paluma' tiveram uma firmeza significativamente maior que os da 'Pedro Sato'. A área injuriada mostrou maior escurecimento e retardo no amarelecimento, indicado pelo maior ângulo Hue, típico do amadurecimento de goiabas. Os valores de cromaticidade foram sempre inferiores nas áreas injuriadas, de ambas as cultivares, indicando menor síntese de pigmentos carotenóides nessas regiões. Os frutos da 'Pedro Sato' foram caracterizados como mais escuros e mais esverdeados que os da 'Paluma' ao longo do período de armazenamento, independentemente das injúrias.
\end{abstract}

Termos para indexação: Psidium guajava, pós-colheita, impacto, compressão, corte, firmeza de polpa, coloração.

\section{EFFECT OF MECHANICAL INJURIES ON FIRMNESS AND COLOR OF 'PALUMA' AND 'PEDRO SATO' GUAVAS}

\begin{abstract}
This work aimed to evaluate the effect of mechanical injuries, impact, compression or cuts on firmness and epidermal color of fruits of two guava cultivars, harvested at mature-green ripeness stage at room temperature. Impact injury was obtained by dropping the guavas twice, from a height of $1.20 \mathrm{~m}$ on a hard surface. Compression injury was done with the fruits submitted to a $3 \mathrm{~kg}$ load for 15 minutes. Cutting fruits obtained cut injury, twice longitudinally to $30 \mathrm{~mm}$ in length and $2 \mathrm{~mm}$ depth. Injured fruits were placed on trays and stored at ambient conditions $\left(23.4 \pm 1{ }^{\circ} \mathrm{C}, 62 \pm 6 \% \mathrm{RH}\right)$. The firmness of fruits submitted to impact and compression injuries was calculated by the relationship of weight $(\mathrm{N})$ /injured area $\left(\mathrm{m}^{2}\right)$. Epidermal color changes were determined by a Minolta $\mathrm{CR}$ $200 \mathrm{~b}$ reflectometer. Daily color measurement was done to compare the color of the injured area with the non-injured area of the same fruit. The compression test indicates that there are no significant differences between the tested cultivars as for flesh firmness. However, 'Paluma' fruits had better firmness than 'Pedro Sato' fruits, when submitted to impact damages. The injured area was darker than intact areas while Hue angle was always higher in these areas, indicating retard in yellowing, typical of guava ripening. Chroma values were always lower in injured areas, for both cultivars. 'Pedro Sato' fruits were characterized as darker and more greenish than 'Paluma' along the storage period, independently of the injuries.
\end{abstract}

Index terms: Psidium guajava, postharvest, impact, compression, cut, flesh firmness, color.

\section{INTRODUÇÃO}

Atualmente, o cultivo da goiabeira (Psidium guajava L.) está sendo desenvolvido em mais de 50 países, das áreas tropicais e subtropicais, incluindo também algumas áreas mediterrâneas, sendo que o Brasil é o segundo maior produtor mundial desta fruta (WWWGH, 2000).

Dentre as causas de perdas pós-colheita das goiabas, destacam-se aquelas devidas à ocorrência de injúrias mecânicas, que podem ser agrupadas em injúrias por impacto, compressão ou corte. Tais injúrias ocasionam danos irreparáveis aos frutos, prejudicando sua qualidade e provocando conseqüente desvalorização comercial. Os danos físicos também alteram as reações bioquímicas normais dos frutos, modificando-lhes a coloração e o sabor, e diminuindo-lhes a vida útil (Chitarra \& Chitarra, 1990).

A suscetibilidade ao dano mecânico é influenciada por vários fatores, como cultivar, grau de hidratação celular, estádio de maturação, tamanho, peso, características epidérmicas e condições ambientais sob as quais os frutos se desenvolveram

1 Trabalho 265/2000. Recebido: 08/12/2000. Aceito para publicação: 30/05/2001.

2 Doutorando do Curso de Pós-Graduação em Produção Vegetal, FCAV-UNESP. Bolsista da FAPESP. bmattiuz@yahoo.com.br

3 Prof. Adjunto do Departamento de Tecnologia, FCAV-UNESP. jfduri@fcav.unesp.br

UNESP - Campus de Jaboticabal, Via de acesso Prof. Paulo Donato Castellane, km 5, 14870-000 Jaboticabal, SP. 
(Wade \& Bain, 1980; Kays, 1991).

Em adição aos típicos sintomas externos e internos, as injúrias mecânicas em frutos são geralmente acompanhadas por elevado número de respostas fisiológicas. Essas injúrias promovem freqüentemente o rompimento das células da epiderme, causando o desenvolvimento de reações enzimáticas e, com isso, o surgimento de compostos de coloração marrom, responsáveis pela depreciação do produto (Radi et al., 1997; Samim \& Banks, 1993). Em tomates, é comum o surgimento de regiões amolecidas, assemelhando-se com acúmulo de água, decorrentes de um manuseio comercial inadequado (Moretti, 1998). Também, de acordo com esse mesmo autor, a ocorrência de impactos menos severos pode não causar sintomatologia externa prontamente observável, no entanto, o efeito acaba repercutindo a "posteriori", produzindo uma injúria interna ("internal bruising").

Objetivou-se avaliar o efeito das injúrias mecânicas por impacto, compressão ou corte na firmeza e na coloração de goiabas 'Paluma' e 'Pedro Sato', armazenadas sob condições ambiente.

\section{MATERIAL E MÉTODOS}

Foram utilizados frutos de goiabeiras das cultivares Paluma e Pedro Sato procedentes do município de Vista Alegre do Alto-SP, colhidos em duas épocas: janeiro (experimento de impacto) e outubro de 1999 (experimentos de compressão e corte).

Depois de colhidos, no estádio de maturação "de vez", correspondente à coloração verde-mate (Pereira, 1995), os frutos foram imediata e cuidadosamente transportados para o Laboratório de Tecnologia dos Produtos Agrícolas da FCAV/ UNESP - Jaboticabal, onde, após imersão em água fria $\left(15^{\circ} \mathrm{C}\right)$ e clorada $\left(150 \mu \mathrm{g}\right.$ de cloro. $\left.\mathrm{L}^{-1}\right)$ por cinco minutos, estes frutos foram submetidos às injúrias mecânicas. Na injúria por impacto, eles foram deixados cair, em queda livre, de uma altura de 1,20 $\mathrm{m}$. Cada fruto sofreu dois impactos na região equatorial, em lados opostos. Para a injúria correspondente à compressão, os frutos foram colocados em um aparelho onde um bloco exercendo um peso de $3 \mathrm{~kg}$ era apoiado, por 15 minutos, provocando 2 lesões em lados opostos e no sentido longitudinal dos frutos. Na injúria por corte, foram realizados dois cortes, em lados opostos, de 30 $\mathrm{mm}$ de comprimento por $2 \mathrm{~mm}$ de profundidade, no sentido longitudinal, usando-se uma lâmina com 1,1 mm de espessura.

As áreas lesionadas foram demarcadas e os frutos acondicionados, em lotes de quatro, em bandejas de isopor e armazenados sob condições de ambiente $\left(23,4 \pm 1{ }^{\circ} \mathrm{C}, 62 \pm 6 \%\right.$ UR).

A firmeza dos frutos foi obtida conforme o proposto por Mohsenin (1986) e Calbo \& Nery (1995), relacionando-se o peso exercido com a área aplanada dos frutos, e pôde ser calculada somente nas injúrias de impacto e de compressão. Os resultados foram expressos em $\mathrm{kPa}$.

Foram efetuadas avaliações diárias da coloração, nas áreas injuriadas e não injuriadas do mesmo fruto, utilizando-se de reflectômetro Minolta CR 200b, onde foram determinados os valores de luminosidade $\left(L^{*}\right), a^{*}$ e $b^{*}$. Esses resultados permitiram calcular o ângulo Hue (cor) e a saturação (Chroma) desta cor, conforme o recomendado por MINOLTA (1994).

\section{RESULTADOS E DISCUSSÃO}

Na Figura 1, consta a firmeza das goiabas 'Paluma' e 'Pedro Sato' sob dois tipos de injúrias mecânicas: impacto e compressão. Verifica-se que, com relação à injúria por compressão, não houve diferença significativa entre as cultivares testadas (média de $61,88 \mathrm{kPa}$ ), mas, com relação ao impacto, os frutos da 'Paluma' tiveram uma firmeza significativamente maior (2,85 kPa) que os da 'Pedro Sato' (2,49 kPa), significando uma maior resistência a esse tipo de dano. Isso pode estar relacionado com as características físicas dos frutos da 'Paluma', que apresentam maior tamanho e peso, além de epiderme mais lisa e pericarpo mais espesso que a 'Pedro Sato' (Kavati, 1997). Esses fatores, conjugados ao grau de hidratação celular, podem ter contribuído para a maior firmeza (Kays, 1991).

Avaliando-se a evolução da luminosidade das áreas lesionadas ao longo do armazenamento (Tabela 1), observa-se que, apesar de não serem detectadas diferenças significativas, tem-se que, ao longo do armazenamento, a área injuriada sempre se apresentou mais escura (decréscimo na luminosidade), em ambas as cultivares e nos três tipos de injúrias. Quando a célula foi rompida, devido à magnitude da força empregada, ocorre o extravasamento do líquido celular e sua conseqüente exposição à ação enzimática, o que promove a oxidação de compostos fenólicos a quinonas, levando ao aparecimento de pigmentos de coloração marrom (Radi et al., 1997). Samim \& Banks (1993) também observaram que a áreas injuriadas de maçãs 'Granny Smith' se tornavam mais escuras que as regiões-controle.

Os frutos da cultivar Pedro Sato apresentam valores médios de $L^{*}$ menores que os da 'Paluma', mostrando uma maior suscetibilidade ao escurecimento, independentemente da injúria. Observa-se, com relação ao tipo de injúria, que o impacto levou a um maior escurecimento aos frutos, para ambas as cultivares. Isso sinaliza que esta lesão é grave, e foi agravada pelo possível rompimento de células por ocasião da injúria.

Na Tabela 2, são mostrados os valores do ângulo Hue (cor) nos frutos da 'Paluma' e da 'Pedro Sato', permitindo notar que a cor, durante o período de armazenamento, evoluiu do verde para o amarelo, corroborando com o observado por Lima (1999). Independentemente da cultivar, os valores de Hue foram sempre maiores nas regiões injuriadas, indicando retardamento no amadurecimento dessas áreas quando comparadas às áreas não injuriadas no mesmo fruto. Isso provavelmente se deve às injúrias impostas nesses frutos, que levaram a uma alteração no processo metabólico normal dessas regiões e, como conseqüência, houve uma irregularidade no amadurecimento conforme o sugerido por Mohsenin (1986) e Chitarra \& Chitarra (1990). As médias obtidas para a cultivar Paluma indicam que as injúrias por compressão e corte influenciaram na cor dos frutos, pois levaram às menores médias.

De acordo com os dados da Tabela 3, observa-se que houve uma tendência de incremento nos valores de cromaticidade ao longo do experimento, independentemente da cultivar ou da injúria. $\mathrm{O}$ aumento na luminosidade, conjugado com o aumento na cromaticidade, revela que, nos frutos, à medida que passavam 
TABELA 1 - Luminosidade $\left(L^{*}\right)$ da parte externa de goiabas 'Pedro Sato' e 'Paluma' submetidas a três tipos de injúrias mecânicas e armazenadas sob condições de ambiente $\left(23,4^{\circ} \mathrm{C}\right.$ e $62 \%$ UR)

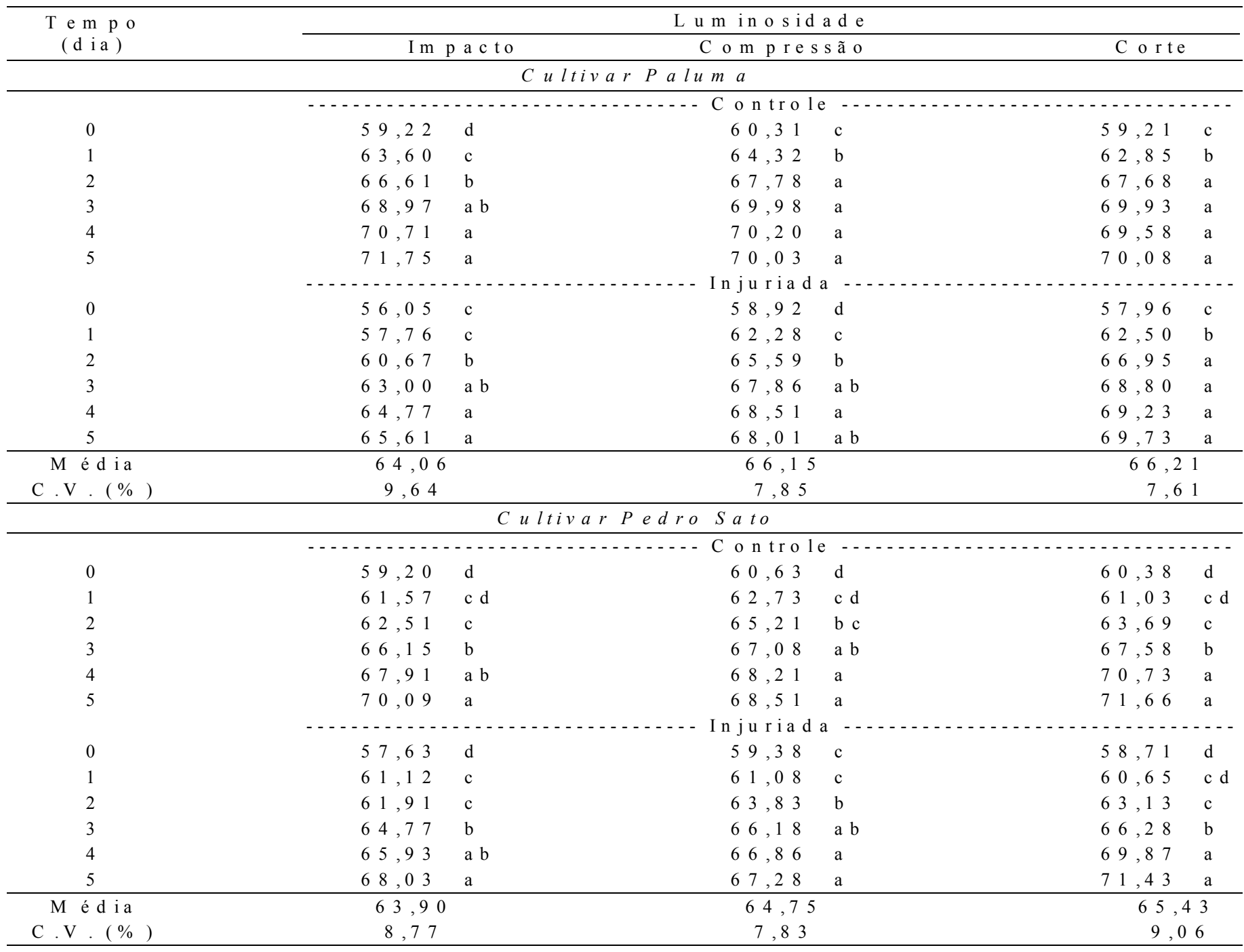

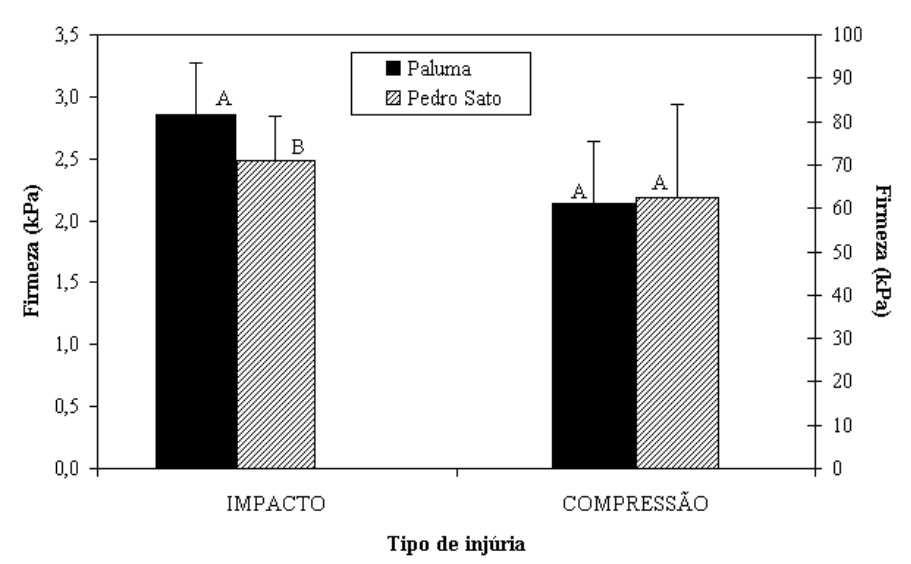

FIGURA 1 - Firmeza dos frutos de duas cultivares de goiabeira quando submetidos a injúrias mecânicas, de impacto e compressão. Colunas com uma mesma letra, dentro de cada injúria, não diferem significativamente entre si, pelo teste de Tukey $(\mathrm{P}<0,05)$. da cor verde para a amarela, ocorria uma redução nos pigmentos verdes (clorofila) e acúmulo nos amarelos (carotenóides). Nas áreas injuriadas, os valores são sempre inferiores ao controle, em ambas as cultivares, indicando uma menor síntese de pigmentos amarelos naquela região. Isto pode influenciar o poder de compra do consumidor, visto que a aparência é um dos fatores mais importantes para a aquisição de um determinado vegetal nas gôndolas dos supermercados.

\section{CONCLUSÕES}

$\mathrm{Na}$ injúria de impacto, os frutos da 'Paluma' tiveram uma firmeza significativamente maior que os da 'Pedro Sato', não observado na injúria de compressão. A área injuriada sempre se mostrou mais escurecida $\left(L^{*}\right)$ que a intacta, assim como houve retardo no amadurecimento indicado pelo maior ângulo Hue, ao longo do armazenamento. Os frutos da cultivar Pedro Sato mostraram-se mais escuros que os da 'Paluma'. 
TABELA 2 - Cor da parte externa (ângulo Hue) de goiabas 'Pedro Sato' e 'Paluma' submetidas a três tipos de injúrias mecânicas e armazenadas sob condições de ambiente $\left(23,4^{\circ} \mathrm{C}\right.$ e $62 \%$ UR)

\begin{tabular}{|c|c|c|c|c|c|c|}
\hline \multirow{2}{*}{$\begin{array}{c}\mathrm{T} \text { e m p o } \\
\text { (d i a ) }\end{array}$} & \multicolumn{6}{|c|}{ ân g u $10 H u e$} \\
\hline & \multicolumn{2}{|c|}{$\mathrm{Im} \mathrm{pacto}$} & \multicolumn{2}{|c|}{ Com pressão } & \multicolumn{2}{|c|}{ Corte } \\
\hline \multicolumn{7}{|c|}{ Cultivar Paluma } \\
\hline & 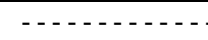 & - & $-\mathrm{Contro}$ & $-\ldots$ & $\ldots \ldots$ & $\cdots$ \\
\hline 0 & 115,87 & $\mathrm{a}$ & 114,77 & $\mathrm{a}$ & 115,35 & $\mathrm{a}$ \\
\hline 1 & 109,98 & $\mathrm{~b}$ & 107,73 & $\mathrm{~b}$ & 107,88 & $\mathrm{~b}$ \\
\hline 2 & 104,89 & $\mathrm{c}$ & 98,22 & $\mathrm{c}$ & 98,56 & $\mathrm{c}$ \\
\hline 3 & 100,11 & $\mathrm{~d}$ & 91,73 & $\mathrm{~d}$ & 92,03 & $\mathrm{~d}$ \\
\hline 4 & 96,22 & $\mathrm{e}$ & 87,67 & $\mathrm{e}$ & 89,83 & $\mathrm{de}$ \\
\hline \multirow[t]{2}{*}{5} & 93,43 & $\mathrm{e}$ & 84,41 & $\mathrm{f}$ & 87,47 & $\mathrm{e}$ \\
\hline & $\ldots$ & - & - Injuriac & $a-$ & $\ldots$ & $\ldots$ \\
\hline 0 & 117,20 & $\mathrm{a}$ & 114,52 & $\mathrm{a}$ & 115,68 & $\mathrm{a}$ \\
\hline 1 & 111,80 & $\mathrm{~b}$ & 108,58 & $\mathrm{~b}$ & 108,84 & $\mathrm{~b}$ \\
\hline 2 & 106,26 & $\mathrm{c}$ & 98,65 & $\mathrm{c}$ & 99,71 & $\mathrm{c}$ \\
\hline 3 & 101,76 & $\mathrm{~d}$ & 92,60 & $\mathrm{~d}$ & 93,47 & $\mathrm{~d}$ \\
\hline 4 & 97,05 & $\mathrm{e}$ & 88,07 & $\mathrm{e}$ & 90,74 & $\mathrm{~d}$ \\
\hline 5 & 94,61 & $\mathrm{e}$ & 84,26 & $\mathrm{f}$ & 87,17 & $\mathrm{e}$ \\
\hline M édia & \multicolumn{2}{|c|}{104,10} & \multicolumn{2}{|c|}{97,60} & \multicolumn{2}{|c|}{98,89} \\
\hline $\mathrm{C} \cdot \mathrm{V} \cdot(\%)$ & \multicolumn{2}{|l|}{9,35} & \multicolumn{2}{|c|}{12,05} & \multicolumn{2}{|c|}{10,67} \\
\hline \multicolumn{7}{|c|}{ Cultivar Pedro Sato } \\
\hline & \multicolumn{6}{|c|}{ - } \\
\hline 0 & 115,70 & $\mathrm{a}$ & 116,42 & a & 116,55 & a \\
\hline 1 & 110,33 & $\mathrm{~b}$ & 114,03 & a & 113,57 & $\mathrm{~b}$ \\
\hline 2 & 107,23 & $\mathrm{c}$ & 107,11 & $\mathrm{~b}$ & 108,17 & $\mathrm{c}$ \\
\hline 3 & 101,25 & $\mathrm{~d}$ & 101,28 & $\mathrm{c}$ & 101,00 & $\mathrm{~d}$ \\
\hline 4 & 96,63 & $\mathrm{e}$ & 95,91 & $\mathrm{~d}$ & 95,80 & $\mathrm{e}$ \\
\hline \multirow[t]{2}{*}{5} & 92,59 & $\mathrm{f}$ & 92,07 & $\mathrm{e}$ & 91,53 & $\mathrm{f}$ \\
\hline & ـ & $-\ldots$ & - Injuriac & 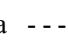 & . & $\ldots$ \\
\hline 0 & 116,35 & $\mathrm{a}$ & 117,01 & $\mathrm{a}$ & 117,74 & $\mathrm{a}$ \\
\hline 1 & 112,23 & $\mathrm{~b}$ & 114,73 & $\mathrm{a}$ & 113,90 & $\mathrm{~b}$ \\
\hline 2 & 110,12 & $\mathrm{~b}$ & 108,67 & $\mathrm{~b}$ & 109,57 & $\mathrm{c}$ \\
\hline 3 & 103,74 & $\mathrm{c}$ & 102,92 & $\mathrm{c}$ & 101,93 & $\mathrm{~d}$ \\
\hline 4 & 98,90 & $\mathrm{~d}$ & 97,40 & $\mathrm{~d}$ & 96,32 & $\mathrm{e}$ \\
\hline 5 & 95,20 & $\mathrm{e}$ & 93,55 & $\mathrm{e}$ & 92,28 & $\mathrm{f}$ \\
\hline M édia & \multicolumn{2}{|c|}{105,02} & \multicolumn{2}{|c|}{105,09} & \multicolumn{2}{|c|}{104,86} \\
\hline $\mathrm{C} . \mathrm{V} \cdot(\%)$ & \multicolumn{2}{|l|}{8,80} & 9,89 & & \multicolumn{2}{|c|}{9.25} \\
\hline
\end{tabular}

\section{REFERÊNCIAS BIBLIOGRÁFICAS}

CALBO, A.G.; NERY, A.A. Medida de firmeza em hortaliças pela técnica de aplanação. Horticultura Brasileira., Brasília, v. 12 , n. 1, p. 14-8, 1995.

CHITARRA, M.I.F.; CHITARRA, A.B. Pós-colheita de frutos e hortaliças: fisiologia e manuseio. Lavras: ESAL-FAEPE, 1990. $320 \mathrm{p}$.

KAVATI, R. Cultivares. In: SIMPÓSIO BRASILEIRO SOBRE A CUlTURA DA GOIABEIRA, 1, 1997. Anais... Jaboticabal:FACV/UNESP-FUNEP-GOIABRÁS, 1997. p. 1-16.

KAYS, J.S. Postharvest physiology of perishable plant products. New York:Van Nostrand Reinhold, 1991. 453 p.

LIMA, M.A. Conservação pós-colheita de goiaba e caracterização tecnológica dos frutos de diferentes genótipos, produzidos em Jaboticabal, SP. 1999. 101 f. Dissertação
(Mestrado em Produção Vegetal) - Faculdade de Ciências Agrárias e Veterinárias, Universidade Estadual Paulista. Jaboticabal, 1999.

MINOLTA. Precise color communication: color control from feeling to instrumentation. Japão, 1994. 49 p.

MOHSENIN, N.N. Physical properties of plant and animal material: structure, physical characteristics and mechanical proprieties. 2. ed. New York: Gordon and Breach, 1986. v. 1, $534 \mathrm{p}$.

MORETTI, C.L. A injúria mecânica de impacto e seus efeitos sobre a fisiologia e a conservação pós-colheita de tomates, 1998. 134 f. Tese (Doutorado em Produção Vegetal) Universidade Federal de Viçosa, Viçosa, 1998.

PEREIRA, F.M. Cultura da goiabeira. Jaboticabal:FUNEP, 1995. $47 \mathrm{p}$. 
TABELA 3 - Cromaticidade (Chroma) da parte externa de goiabas 'Pedro Sato' e 'Paluma' submetidas a três tipos de injúrias mecânicas e armazenadas sob condições de ambiente $\left(23,4^{\circ} \mathrm{C}\right.$ e $62 \%$ UR)

\begin{tabular}{|c|c|c|c|c|c|c|}
\hline \multirow{2}{*}{$\begin{array}{l}\text { T e m po } \\
(\mathrm{d} \mathrm{i} \mathrm{a})\end{array}$} & \multicolumn{6}{|c|}{ Crom aticidade } \\
\hline & \multicolumn{2}{|c|}{$\mathrm{Im} \mathrm{p} \mathrm{a} \mathrm{c} \mathrm{to}$} & \multirow{2}{*}{\multicolumn{2}{|c|}{$\begin{array}{l}\mathrm{C} \text { o } \mathrm{m} \text { p ressão } \\
l u m a\end{array}$}} & \multicolumn{2}{|c|}{ Corte } \\
\hline \multicolumn{5}{|c|}{ Cultivar P alu $m$ a } & & \\
\hline & - & $\cdots$ & - C o n tro & & - & - - \\
\hline 0 & 44,98 & $\mathrm{c}$ & 44,22 & $\mathrm{~b}$ & 46,15 & $\mathrm{~b}$ \\
\hline 1 & 48,78 & $\mathrm{~b}$ & 47,38 & $\mathrm{a}$ & 49,85 & $\mathrm{a}$ \\
\hline 2 & 50,60 & $\mathrm{ab}$ & 49,17 & $\mathrm{a}$ & 51,65 & $\mathrm{a}$ \\
\hline 3 & 51,28 & $\mathrm{ab}$ & 48,81 & a & 51,25 & a \\
\hline 4 & 51,60 & $\mathrm{ab}$ & 48,83 & $\mathrm{a}$ & 49,94 & a \\
\hline \multirow[t]{2}{*}{5} & 51,93 & a & 47,94 & $\mathrm{a}$ & 49,48 & a \\
\hline & -- & -- & - In juriac & & $-\ldots \ldots$ & 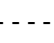 \\
\hline 0 & 42,36 & $\mathrm{c}$ & 42,87 & $\mathrm{~b}$ & 46,15 & $\mathrm{~b}$ \\
\hline 1 & 44,15 & b c & 45,76 & a & 49,00 & $\mathrm{a} b$ \\
\hline 2 & 46,10 & $\mathrm{a} \mathrm{b}$ & 46,77 & a & 50,77 & a \\
\hline 3 & 47,67 & a & 47,27 & a & 50,68 & a \\
\hline 4 & 47,98 & a & 46,79 & a & 49,08 & a \\
\hline 5 & 48,55 & a & 46,15 & $\mathrm{a}$ & 48,25 & $\mathrm{ab}$ \\
\hline M é d i a & \multicolumn{2}{|l|}{48,00} & \multicolumn{2}{|c|}{46,83} & \multicolumn{2}{|c|}{49,35} \\
\hline $\mathrm{C} . \mathrm{V} \cdot(\%)$ & \multicolumn{2}{|l|}{8,93} & \multicolumn{2}{|l|}{6,86} & \multicolumn{2}{|c|}{6,38} \\
\hline \multicolumn{7}{|c|}{$C$ ultivar $P$ edro $S$ a to } \\
\hline & 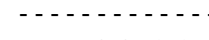 & $-\cdots$ & - - C o n tro & -- & $----\cdot$ & -- \\
\hline 0 & 44,38 & d & 41,12 & $\mathrm{c}$ & 42,56 & d \\
\hline 1 & 46,47 & $\mathrm{~cd}$ & 43,63 & $\mathrm{~b} \mathrm{c}$ & 44,29 & c d \\
\hline 2 & 46,94 & $\mathrm{bc} \mathrm{d}$ & 45,62 & $\mathrm{a} b$ & 46,49 & $\mathrm{~b} c \mathrm{c}$ \\
\hline 3 & 49,32 & $\mathrm{abc}$ & 46,23 & a & 48,27 & $\mathrm{a} b$ \\
\hline 4 & 49,91 & $\mathrm{ab}$ & 47,01 & a & 49,79 & a \\
\hline \multirow[t]{2}{*}{5} & 51,94 & a & 47,66 & a & 50,22 & a \\
\hline & 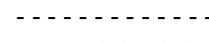 & $\cdots$ & - Injuriac & - & ----- & $\cdots$ \\
\hline 0 & 42,68 & $\mathrm{c}$ & 41,51 & d & 41,30 & e \\
\hline 1 & 44,98 & b c & 43,28 & c d & 44,11 & $\mathrm{~d} \mathrm{e}$ \\
\hline 2 & 45,63 & b c & 45,39 & b c & 45,87 & $\mathrm{c} \mathrm{d}$ \\
\hline 3 & 47,28 & $\mathrm{ab}$ & 46,74 & $a b$ & 47,35 & $\mathrm{~b} \mathrm{c}$ \\
\hline 4 & 47,78 & $\mathrm{a} \mathrm{b}$ & 47,40 & $a b$ & 49,98 & $\mathrm{a} b$ \\
\hline 5 & 49,80 & $\mathrm{a}$ & 47,76 & $\mathrm{a}$ & 51,09 & $\mathrm{a}$ \\
\hline M é d ia & \multicolumn{2}{|l|}{47,26} & \multicolumn{2}{|c|}{45,28} & \multicolumn{2}{|c|}{46,78} \\
\hline C.V. $(\%)$ & \multicolumn{2}{|l|}{7,78} & 7,61 & & \multicolumn{2}{|c|}{8,26} \\
\hline
\end{tabular}

* Para cada cultivar, médias seguidas por uma mesma letra não diferem significativamente entre si, pelo teste de Tukey $(\mathrm{P}<0,05)$

RADI, M.; MAHROUZ, M.; JAOUAD, A. Phenolic composition, browning susceptibility, and carotenoid content of several apricot cultivars at maturity. HortScience, Alexandria, v. 32, n. 6, p. 1087-1091. 1997.

SAMIM, W.; BANKS, N.H. Colour changes in bruised apple fruit tissue. New Zealand Journal of Crop and Horticultural Science, Wellington, v. 21, n. 4, p. 367-72, 1993.
WADE, N.L.; BAIN, J.M. Physiological and anatomical studies of surface pitting of sweet cherry fruit in relation to bruising, chemical treatments and storage conditions. Journal of Horticultural Science, Kent, v. 55, n. 4, p. 375-384, 1980.

WWWGH. Welcome to the Wonderful World of Guavas Homepage. Distribution. Disponível em <http:// www.ocf.berkeley.edu/ montymex/guava/guavaintro.html>. Acessado em 29 nov. 2000. 